\title{
Exploring links between Arctic amplification and mid-latitude weather
}

\author{
James A. Screen ${ }^{1,2}$ and Ian Simmonds ${ }^{1}$ \\ Received 12 December 2012; revised 15 January 2013; accepted 16 January 2013.
}

[1] This study examines observed changes (1979-2011) in atmospheric planetary-wave amplitude over northern mid-latitudes, which have been proposed as a possible mechanism linking Arctic amplification and mid-latitude weather extremes. We use two distinct but equally-valid definitions of planetary-wave amplitude, termed meridional amplitude, a measure of north-south meandering, and zonal amplitude, a measure of the intensity of atmospheric ridges and troughs at $45^{\circ} \mathrm{N}$. Statistically significant changes in either metric are limited to few seasons, wavelengths, and longitudinal sectors. However in summer, we identify significant increases in meridional amplitude over Europe, but significant decreases in zonal amplitude hemispherically, and also individually over Europe and Asia. Therefore, we argue that possible connections between Arctic amplification and planetary waves, and implications of these, are sensitive to how waves are conceptualized. The contrasting meridional and zonal amplitude trends have different and complex possible implications for midlatitude weather, and we encourage further work to better understand these. Citation: Screen, J. A., and I. Simmonds (2013), Exploring links between Arctic amplification and mid-latitude weather, Geophys. Res. Lett., 40, doi:10.1002/grl.50174.

\section{Introduction}

[2] One of the clearest manifestations of climate change is greater near-surface warming of the high latitudes than the low or middle latitudes of the Northern Hemisphere. This so-called Arctic amplification (AA) is evident in observations over recent decades [Screen and Simmonds, 2010; Serreze and Barry, 2011] and is a consistent feature in climate model simulations, which include increases in greenhouse gas concentrations [Solomon et al., 2007]. It follows from its definition that AA is associated with a reduction of the nearsurface meridional (north-south) temperature gradient.

[3] Recently, Francis and Vavrus [2012] (hereafter FV12) suggested that a relaxation of the meridional thickness (temperature) gradients over the last three decades has led to (a) weakened zonal winds and (b) greater north-south meandering of the mid-latitude circulation over North America and the Atlantic Ocean. In support of (b), FV12 identified a statistically significant increase in the maximum latitude of selected $500 \mathrm{hPa}$ geopotential height $\left(Z_{500}\right)$ isopleths, which

\footnotetext{
${ }^{1}$ School of Earth Sciences, University of Melbourne, Melbourne, Victoria, Australia.

${ }^{2}$ College of Engineering, Mathematics and Physical Sciences, University of Exeter, Exeter, UK.

Corresponding author: J. Screen, College of Engineering, Mathematics and Physical Sciences, Harrison Building, Streatham Campus, University of Exeter, North Park Road, Exeter, Devon, EX4 4QF, UK. (j.screen@exeter.ac.uk)

C 2013 . American Geophysical Union. All Rights Reserved. 0094-8276/13/10.1002/grl.50174
}

they interpreted as an elongation of the meanders and an increase in north-south wave amplitude. The authors further postulated that larger amplitude waves propagate more slowly (than smaller amplitude waves) and, therefore, favor persistent weather conditions such as heat waves, cold spells, droughts, and floods. The argument presented by FV12 potentially provides a causal link between AA and extreme weather in mid-latitudes and may, if robust, help reconcile the ostensibly large number of such weather events in the last decade [Coumou and Rahmstorf, 2012], with significant environmental, economic and societal implications.

[4] Liu et al. [2012] invoke a somewhat similar mechanism to link Arctic sea ice loss to anomalous snowfall across the United States (US), Europe, and East Asia. These authors suggest that Arctic sea ice loss has induced wintertime tropospheric circulation anomalies with pronounced meridional meanders in mid-latitudes. They further suggest that this circulation change has resulted in more frequent episodes of atmospheric blocking. Atmospheric blocking is a key driver of extreme weather in the mid-latitudes [e.g., Buehler et al., 2011; Sillmann et al., 2011; Pfahl and Wernli, 2012]. Other authors have also proposed links between Arctic sea ice loss and mid-latitude cold extremes in a number of regions, including northern Europe and Asia [Honda et al., 2009; Petoukhov and Semenov, 2010; Overland et al., 2011; Inoue et al., 2012]. On this potential linkage, Hopsch et al. [2012] concluded "that whilst suggested pathways in previously published studies seem reasonable [...], our [observational] results show that these are not yet robust enough from a statistical significance perspective." Some modeling studies support a causal link between AA and mid-latitude weather, while others do not (see, e.g., Discussion in Screen et al. [2013]). Thus, whether or not AA drives robust and observable mid-latitude climate change remains an open question.

[5] In this manuscript, we present new evidence and insight into the changes in mid-latitude (Rossby) waves over recent decades. We expand on FV12 in three key ways. First, we use a different approach to measuring planetarywave amplitude and consider two alternative definitions of amplitude that reflect different aspects of the mid-latitude circulation. Second, we examine wave amplitude changes at all longitudes, and also over three separate longitudinal sectors, whereas FV12 only examined longitudes $0^{\circ} \mathrm{W}-140^{\circ} \mathrm{W}$. This enables identification of possible wave amplitude changes and their potential impacts over, e.g., Europe and Asia. Third, we explicitly separate different wavelengths to determine if waves of particular spatial scales are exhibiting significant changes. This also enables identification of any trends in wavelength, which is directly related to wave propagation speed.

\section{Data and Methods}

[6] We employ Fourier decomposition to characterize the waves in the mid-latitude mid-tropospheric flow, and changes 
therein. Using the fast Fourier transform (FFT), we express the longitudinal structure of variable $y$ as,

$$
y=A_{0}+\sum_{m=1}^{n / 2} A_{m} \cdot \sin \left(m x-\varphi_{m}\right),
$$

where $x, A, \varphi$, and $m$ are the longitudinal distance, wave amplitude, phase, and wavenumber, respectively. We apply the FFT in two different frameworks, which correspond to different ways of defining and conceptualizing the waves in the circulation. Both approaches utilize daily $(00 \mathrm{z}) Z_{500}$ from the European Centre for Medium-range Weather Forecasts' ERA-Interim reanalysis [Dee et al., 2011] for the period 1 January 1979 to 31 December 2011. Over mid-latitudes the reanalysis is relatively well-constrained by soundings and, because we limit our analyses to the period post-1979, by satellite retrievals. This largely circumvents issues relating to the accuracy of reanalyses in datasparse regions and prior to the assimilation of satellite data. The results presented are not sensitive to this choice of reanalysis and almost identical results were found with the National Centers for Environmental Prediction-National Center for Atmospheric Research reanalysis (the data set used by FV12).

[7] In the first framework, we define the waves in terms of the latitude of selected $Z_{500}$ isopleths. In this framework, wave amplitude describes the meridional extent of the meanders in the flow, hereafter referred to as "meridional amplitude." The isopleths used were 5400, 5500, 5700, and $5600 \mathrm{~m}$ for winter (January-February-March; JFM), spring (April-May-June; AMJ), summer (July-August-September; JAS), and autumn (October-November-December; OND), respectively. These selected isopleths and seasonal definitions are consistent with FV12. We calculated the latitude of the selected $Z_{500}$ isopleth (denoted $\theta_{\text {iso }}$ ) at each longitude. Because the selected isopleth commonly lies between grid points, $\theta_{\text {iso }}$ was approximated by linear interpolation from the neighboring grid points. For longitudes where there were multiple instances of the selected isopleth, the most southerly intersection was chosen.

[8] In the second framework, we define the waves in terms of the $Z_{500}$ around the $45^{\circ} \mathrm{N}$ latitude circle. In this framework, wave amplitude describes the height and depth of ridges and troughs around $45^{\circ} \mathrm{N}$, hereafter referred to as "zonal amplitude." In what follows we are careful to use the colloquial terms "ridge" and "trough" only in the context of zonal waves and "meander" only in the context of meridional waves. The two measures of amplitude reflect different characteristics of the mid-latitude circulation and changes therein, and have differing implications for mid-latitude weather, which will be discussed later.

[9] Fourier decomposition was also undertaken for four longitudinal sectors. The first of these covers the sector $0^{\circ} \mathrm{W}-140^{\circ} \mathrm{W}$, which includes North America and the Atlantic Ocean (NAmAtl) and is the same as used by FV12. The other three sectors are $60^{\circ} \mathrm{E}-60^{\circ} \mathrm{W}, 60^{\circ} \mathrm{W}-180^{\circ} \mathrm{W}$ and $60^{\circ} \mathrm{E}-180^{\circ} \mathrm{E}$, which encompass Europe and the Atlantic Ocean (EuroAtl), North America and the western Pacific Ocean (NAmWP), and Asia and the eastern Pacific Ocean (AsiaEP), respectively. To conduct the FFT on these longitude sectors, we assume periodic conditions and an additional point was added at the eastern limit $(n+1$, where $n$ is the number of longitude points) with a value equal to that at the western limit $(n=1)$. In this regional framework, the wavelength of the various wavenumbers is reduced proportionally from those in the hemispheric case. For clarity in the text, we provide the corresponding wavelength (at $45^{\circ} \mathrm{N}$ ) in parentheses.

[10] In all frameworks, the FFT was first performed on daily $Z_{500}$ data to yield daily amplitudes, which were subsequently averaged by season. We analyze seasonal-mean time-series of meridional amplitude and zonal amplitude for the first ten zonal wavenumbers (denoted $A_{\mathrm{M}, m}$ and $A_{\mathrm{Z}, m}$ where $m$ is the wavenumber), which explain the vast majority of variance in the Northern Hemisphere mid-latitude circulation. We also present meridional amplitude and zonal amplitude for the first 10 wavenumbers combined (denoted $A_{\mathrm{M}}$ and $A_{\mathrm{Z}}$ ), calculated as,

$$
A_{\mathrm{M}}=\sqrt{\sum_{m=1}^{m=10} A_{\mathrm{M}, m}^{2}} \text { and } A_{Z}=\sqrt{\sum_{m=1}^{m=10} A_{\mathrm{Z}, m}^{2}}
$$

[11] $A_{\mathrm{M}}$ and $A_{\mathrm{Z}}$ closely approximate the spatial standard deviation and can be considered measures of total "waviness" in the meridional and zonal frameworks, respectively. To facilitate comparison between meridional amplitude and zonal amplitude, and between different wavelengths, all time-series have been normalized by removing their mean and dividing by their standard deviation $(\sigma)$.

[12] Trends in seasonal-mean meridional amplitude and zonal amplitude were calculated by least-squares linear regression for the 33 year period 1979-2011 and are expressed in units of $\sigma$ per decade. We have tested the trends for statistical significance, against a null hypothesis of zero trend, at the $p \leq 0.05$ level using a two-tailed Student's $t$-test.

\section{Results}

[13] Figure 1 illustrates the applicability of the wave decomposition method to characterize the mid-tropospheric circulation. Figure 1a shows the $Z_{500}$ field from the 7 January 2010. This particular day, and winter 2009/2010 in general, was characterized by anomalously cold conditions in the United Kingdom and northwestern mainland Europe [Cattiaux et al., (2010]. An outbreak of cold polar air is clearly visible in Figure 1a as a pronounced trough (southward meander) in the $Z_{500}$ field extending from the central Arctic to northern France. The eastern coast of the US was also anomalously cold during this winter [Guirguis et al., 2011] and can be seen to lie under a trough (southward meander) in Figure 1a. Although winter 2009/2010 hit the headlines for the frigid conditions in Europe and the eastern US, other mid-latitude regions experienced anomalous warmth [Guirguis et al., 2011], for example, western Canada, which lies under a pronounced ridge (northward meander) in Figure 1a. This neatly illustrates how waves in the $Z_{500}$ field are of fundamental importance to mid-latitude weather. Schubert et al. [2011] discuss other examples of mid-latitude temperature and precipitation extremes that were related to well-developed Rossby wave patterns.

[14] The longitudinal structure of $Z_{500}$ around $45^{\circ} \mathrm{N}$ on 7 January 2010 is shown by the dotted blue line in the top panel of Figure $1 \mathrm{~b}$ and the longitudinal structure of $\theta_{\text {iso }}$ by the dotted black line. The second to sixth rows of Figure $1 \mathrm{~b}$ show the wave components, from zonal wavenumber 1 $(28,334 \mathrm{~km})$ to wavenumber $5(5667 \mathrm{~km})$, derived from the two decomposition methods. On this day, and at any 

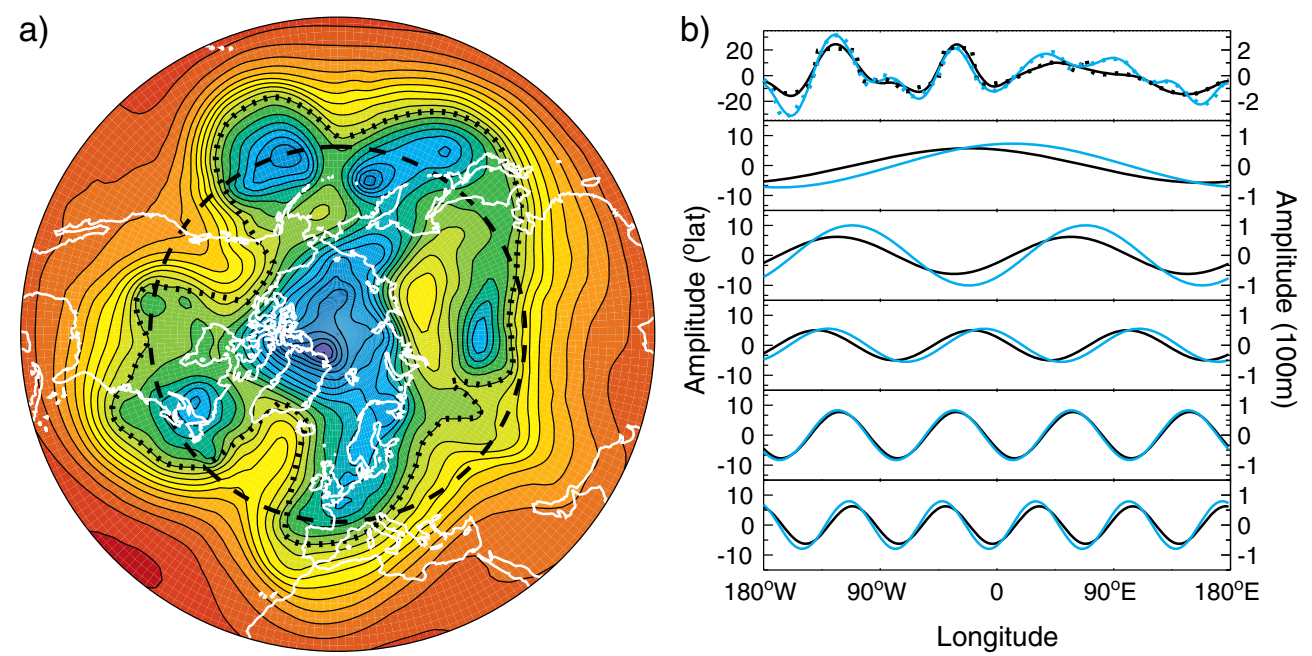

Figure 1. (a) $500 \mathrm{hPa}$ geopotential heights $\left(Z_{500}\right)$ for 7 January 2010 . Contours are drawn at intervals of $50 \mathrm{~m}$. The dotted line denotes the approximate latitude of the $5400 \mathrm{~m}$ isopleth $\left(\theta_{\text {iso }}\right)$. The dashed black line marks the $45^{\circ} \mathrm{N}$ latitude circle. (b) The top panel shows the longitudinal structure of $\theta_{\text {iso }}$ (black dotted line, zonal-mean removed) and $Z_{500}$ at $45^{\circ} \mathrm{N}$ (blue dotted line, zonal-mean removed), and the sum of the first 10 wave components for the decomposition of $\theta_{\text {iso }}$ (black solid) and $Z_{500}$ (blue solid). The second to sixth rows show the wave components for zonal wavenumbers 1 to 5 , respectively, for the decomposition of $\theta_{\text {iso }}$ (black) and $Z_{500}$ (blue). In all panels, the black lines are plotted against the left vertical axis and the blue lines against the right vertical axis.

other point in time, the circulation reflects the superposition of various wavenumbers differing in amplitude and phase. The sum of the first 10 waves is shown by the solid lines in the top panel of Figure $1 \mathrm{~b}$, and is a very close approximation to the real $\theta_{\text {iso }}$ and $Z_{500}$ (dotted lines). The first 10 waves explain $94 \%$ of the longitudinal variance of $\theta_{\text {iso }}$ and $98 \%$ of the longitudinal variance of $Z_{500}$. Considering all days, the first 10 wave components explain on average $90 \%$ of the daily longitudinal variance of $\theta_{\text {iso }}$ and $97 \%$ of the daily longitudinal variance of $Z_{500}$.

[15] Figure 2a shows linear trends from 1979 to 2011 in seasonally-averaged meridional amplitude. Statistically significant trends (at the $p \leq 0.05$ level) are identified by the black dots. The trends in $A_{\mathrm{M}}$ are positive in JAS and OND, and

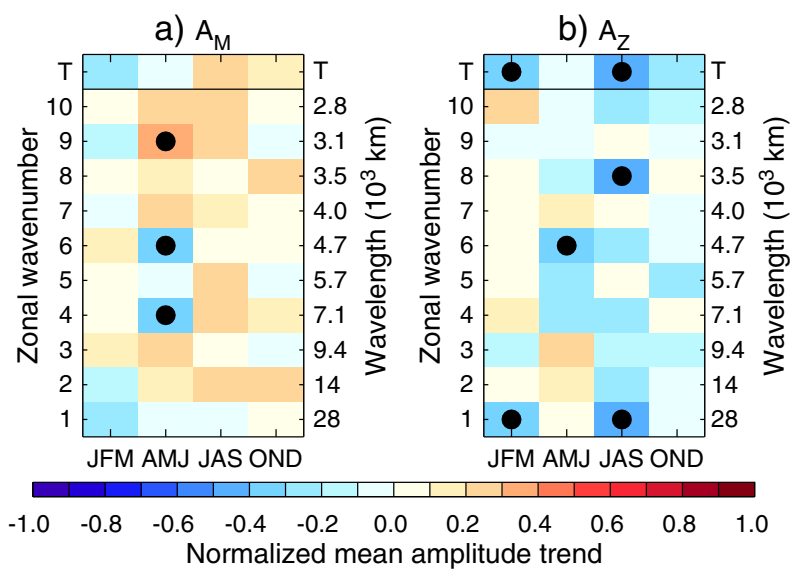

Figure 2. (a) Trends in meridional amplitude as a function of season and wavelength for the hemispheric domain. "T" denotes the combination of wavenumbers 1 to $10\left(A_{\mathrm{M}}\right)$. Trends significant at the $p \leq 0.05$ level are shown by black dots. The units are $\sigma$ per decade. (b) Same as Figure 2a, but for zonal amplitude. negative in JFM and AMJ, but none of these are significant. Only three of the trends for individual wavelengths are statistically significant. These correspond to an increase in $A_{\mathrm{M}, 9}$ $(3148 \mathrm{~km})$ and decreases in $A_{\mathrm{M}, 4}(7083 \mathrm{~km})$ and $A_{\mathrm{M}, 6}$ $(4722 \mathrm{~km})$ in AMJ. We note that this number of significant trends ( $7 \%$ of 40 trends considered) is not appreciably larger than would be expected by chance alone $(5 \%)$. Ignoring the lack of significance, there is weak general tendency toward larger $A_{\mathrm{M}, m}$ (70\% positive trends and $30 \%$ negative trends).

[16] Figure 2b shows the linear trends in seasonally averaged zonal amplitude. Negative $A_{Z}$ trends are found in all seasons, although only the JFM and JAS trends are significant. The significant $A_{\mathrm{Z}}$ decrease in JAS is in stark contrast to the $A_{\mathrm{M}}$ increase in this season. Considering the wavelengths individually, only four significant zonal amplitude trends are found. These correspond to reductions in $A_{\mathrm{Z}, 1}$ $(28,334 \mathrm{~km})$ in JFM, $A_{Z, 6}(4722 \mathrm{~km})$ in AMJ, and $A_{\mathrm{Z}, 1}$ $(28,334 \mathrm{~km})$ and $A_{Z, 8}(3542 \mathrm{~km})$ in JAS. Again, this number of significant trends ( $10 \%$ of 40 trends considered) is not appreciably larger than would be expected by chance alone. Even ignoring the lack of significance, there is no clear tendency toward larger or smaller $A_{\mathrm{Z}, m}$ (60\% negative trends and $40 \%$ positive trends). Nor is there a general tendency toward longer or shorter wavelengths (i.e., longer wavelengths are not increasing in zonal amplitude at the expense of shorter wavelengths), or vice versa. However, we note that the majority of the significant trends in both meridional and zonal amplitude have occurred outside the range of zonal wavenumbers typically associated with (quasi-) stationary Rossby waves $(m=2-5)$. Recall that the zonal amplitude is defined at $45^{\circ} \mathrm{N}$. The main findings that relatively few trends are significant and that the trends are not predominately in one direction also apply if other latitudes are chosen (e.g., $40^{\circ} \mathrm{N}$ and $50^{\circ} \mathrm{N}$; not shown).

[17] Figure 3 shows the meridional amplitude $(\mathrm{a}-\mathrm{d})$ and zonal amplitude $(\mathrm{e}-\mathrm{h})$ trends for four longitudinal sectors. Here we show only individual trends for zonal wavenumbers 


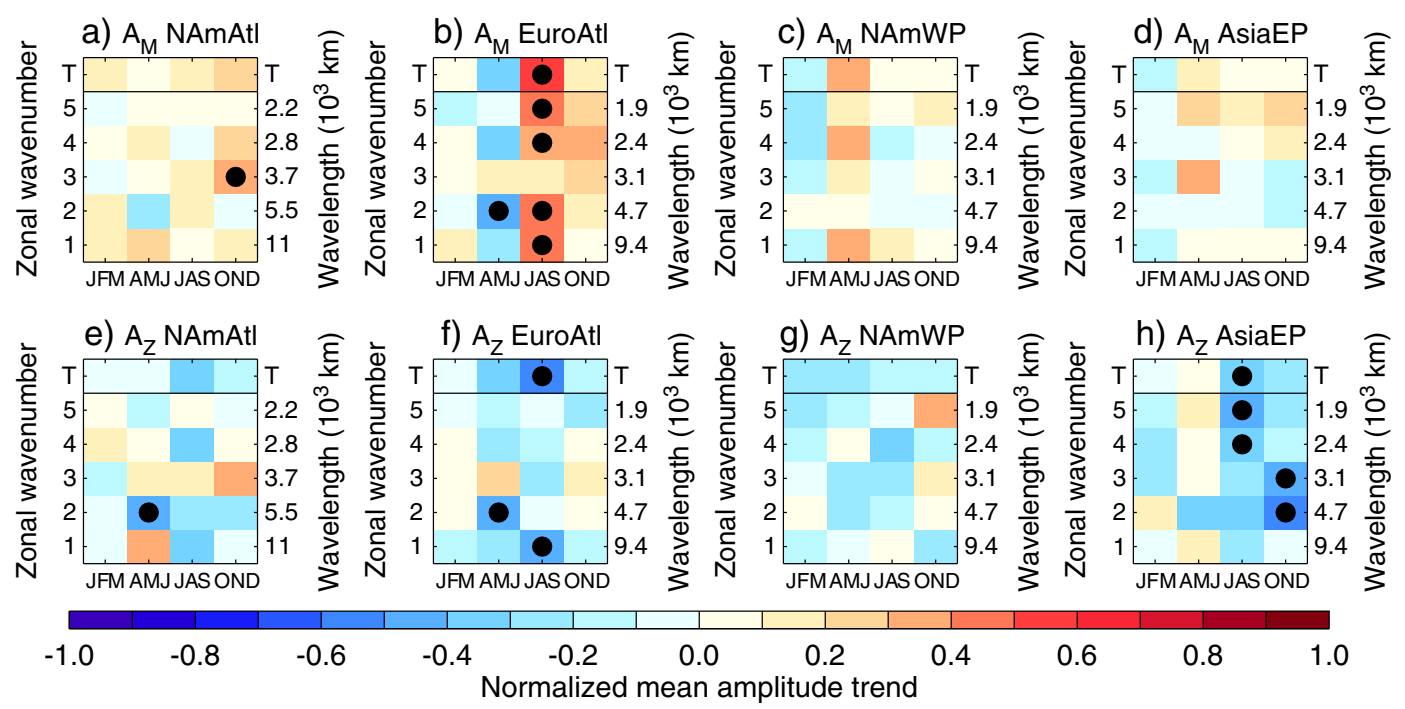

Figure 3. Trends in meridional amplitude as a function of season and wavelength for the regional domains of (a) $0^{\circ} \mathrm{W}-140^{\circ} \mathrm{W}$, (b) $60^{\circ} \mathrm{W}-60^{\circ} \mathrm{E}$, (c) $60^{\circ} \mathrm{E}-180^{\circ} \mathrm{E}$, and (d) $60^{\circ} \mathrm{W}-180^{\circ} \mathrm{W}$. " $\mathrm{T}$ " denotes the combination of wavenumbers 1 to $10\left(A_{\mathrm{M}}\right)$. Trends significant at the $p \leq 0.05$ level are shown by black dots. The units are $\sigma$ per decade. (e-h) Same as Figures $3 \mathrm{a}-3 \mathrm{~d}$, but for zonal amplitude.

$1-5$, as in the regional framework wavenumbers greater than 5 correspond to short wavelengths with small (negligible) amplitude. At this regional scale, wavenumbers larger than 1-2 are likely to reflect synoptic-scale propagating waves and not (quasi-)stationary Rossby waves. For brevity in the text, we will focus on the $A_{\mathrm{M}}$ and $A_{\mathrm{Z}}$ trends and only refer to the trends for individual wavelengths in the few cases where the number of these trends that are significant is appreciably larger than would be expected by chance.

[18] Over NAmAtl, $A_{\mathrm{M}}$ increases and $A_{\mathrm{Z}}$ decreases are found in all seasons, but none achieve statistical significance. The $A_{\mathrm{M}}$ increases are consistent with $\mathrm{FV} 12$, as is the finding that the largest $A_{\mathrm{M}}$ increase has occurred in OND. However, these $A_{\mathrm{M}}$ trends are not statistically significant at the $p \leq 0.05$ (or even at the 0.1) level. $A_{Z}$ has decreased in all seasons, but not significantly in any season. Over EuroAtl, $A_{\mathrm{M}}$ has increased significantly in JAS. Significant increases are also identified for $A_{\mathrm{M}, 1}(9445 \mathrm{~km}), A_{\mathrm{M}, 2}$ $(4722 \mathrm{~km}), A_{\mathrm{M}, 4}(2361 \mathrm{~km})$, and $A_{\mathrm{M}, 5}(1889 \mathrm{~km}) . A_{\mathrm{M}}$ has increased in $\mathrm{OND}$, as has $A_{\mathrm{M}, m}$ of all individual wavelengths considered, but none of these are significant. $A_{Z}$ has decreased in all seasons, but only significantly in JAS. Note that the $A_{\mathrm{M}}$ and $A_{\mathrm{Z}}$ trends are both significant in JAS over EuroAtl, but of opposite sign. No significant trends are found over NAmWP. No significant meridional amplitude trends are found over AsiaEP, but $A_{Z}$ has decreased significantly in JAS. In this season, zonal amplitude decreases are identified for all wavelengths considered and are significant for $A_{Z, 4}(2,361 \mathrm{~km})$ and $A_{\mathrm{Z}, 5}(1,889 \mathrm{~km})$. Although the $A_{\mathrm{Z}}$ decrease in OND is not significant, all wavelengths considered display zonal amplitude decreases in this season and two are significant, $A_{\mathrm{Z}, 2}(4722 \mathrm{~km})$ and $A_{\mathrm{Z}, 3}(3148 \mathrm{~km})$.

\section{Discussion}

[19] At first glance, the opposing meridional and zonal amplitude trends in some seasons and sectors may seem at odds. However, it is plausible, and in what follows we argue it is even to be expected, that the two measures of amplitude show differing changes.

[20] Figure 4a shows an idealized $Z_{500}$ field, increasing from north to south and with a wave-2 disturbance. Now suppose that AA acts on this field. Figure $4 \mathrm{~b}$ shows the a)

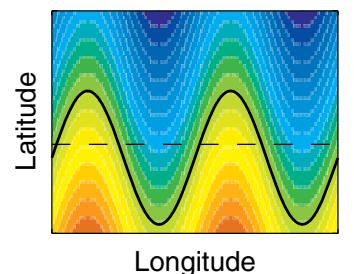

c)

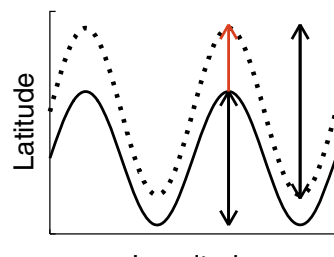

Longitude b)

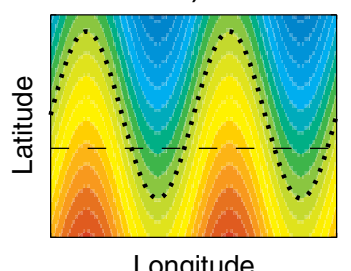

d)

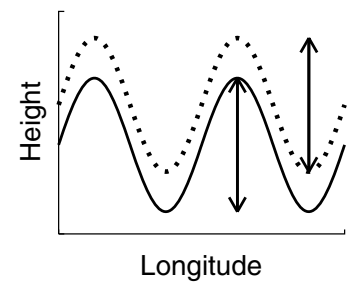

Figure 4. (a) An idealized $500 \mathrm{hPa}$ geopotential height $\left(Z_{500}\right)$ field, with increasing $Z_{500}$ from north to south and a wave-2 disturbance. An arbitrary isopleth is highlighted. (b) The corresponding $Z_{500}$ field after the $Z_{500}$ at all longitudes is increased by an arbitrary amount that scales with latitude, to mimic the effects of Arctic amplification (AA), with the same isopleth highlighted as in Figure 4a. (c) A horizontal cross-section showing the highlighted isopleths from Figures $4 \mathrm{a}$ and $4 \mathrm{~b}$, as solid and dotted lines, respectively. (d) A vertical cross-section showing the $Z_{500}$ along the dashed lines in Figures $4 \mathrm{a}$ and $4 \mathrm{~b}$, as solid and dotted lines, respectively. In Figure 4c, the meridional amplitude increases in response to AA whereas in Figure 4d, the zonal amplitude remains constant, as indicated by the black arrows. 
resultant $Z_{500}$ field when the $Z_{500}$ at all longitudes is increased by an amount that scales with latitude (in this idealized case, the increase is larger at the northern limit than at the southern limit by a factor of 3 , which decreases the north-south $Z_{500}$ gradient by $20 \%$ ). Figure $4 \mathrm{c}$ shows the meanders defined by a selected isopleth in Figure 4a and the same isopleth in Figure $4 \mathrm{~b}$. In response to AA the meanders shift poleward, but not equally at all longitudes. This reflects that the northward meanders are located in a region of larger $Z_{500}$ increase than the southward meanders, and hence the former shift poleward more than the latter. Thus, meridional amplitude increases in the presence of AA. Figure $4 d$ shows the waves defined by sampling $Z_{500}$ along a line of latitude (dashed lines in Figures $4 a$ and $4 b$ ). In this case, the wave shifts equally at all longitudes and zonal amplitude remains unchanged. Furthermore, it is possible for zonal amplitude to decrease at all latitudes and for meridional amplitude to still increase, if the influence of AA is greater than the influence of decreased zonal amplitude. We propose that this scenario explains the opposing trends in meridional and zonal amplitude in some seasons and sectors.

[21] Of course in reality, meridional amplitude is affected by multiple influences other than AA and it is unsurprising that $A_{\mathrm{M}}$ increases are not identified, or not found to be significant, in all seasons and sectors. In fact, it is notable that relatively few seasons, wavelengths, or longitudinal sectors show significant meridional amplitude trends. We argue therefore, that while meridional amplitude increases are consistent with AA, the changes in meridional amplitude over recent decades are relatively small compared to the year-to-year variability. This partly reflects that AA is weaker at $500 \mathrm{hPa}$ than near the surface [e.g., Screen and Simmonds, 2010; Screen et al., 2012]. It is also worth noting that $\mathrm{AA}$ at $500 \mathrm{hPa}$ is likely driven by fundamentally different processes than AA in the lowermost atmosphere. Although lower tropospheric AA has been predominantly driven by Arctic sea ice loss [Screen et al., 2012], there is no evidence of Arctic sea ice loss causing significant change in the meridional temperature gradient at $500 \mathrm{hPa}$ [see, e.g., Screen et al., 2013, Figure 6]. Instead, Arctic warming aloft appears to be primarily remotely-driven by sub-Arctic sea surface temperature changes [Screen et al., 2012]. Therefore, we argue that the meridional amplitude increases are likely not caused by Arctic sea ice loss alone, and that is it important to differentiate the atmospheric impacts of AA, which is driven by multiple processes including sea ice loss, from the impacts of solely Arctic sea ice loss. It is important to note also that links between planetary-wave amplitude and AA are unlikely to be one-way, as changes in wave amplitude impact poleward energy transport and therefore, may be a cause of AA as well as a consequence of it.

[22] It is of interest to compare our results to those of FV12 for the case of meridional amplitude over the NAmAtl region. We find statistically insignificant positive trends in all seasons, in contrast to the comparatively larger (and significant) increases in JAS and OND suggested by FV12. These differences appears to relate to the precise metric analyzed and can be understood using the idealized example above. FV12 effectively measure the poleward shift of the most northerly point on the wave (marked by the red arrow in Figure 4c) which is larger than the change in meridional amplitude (difference between the two black arrows in
Figure 4c). Thus, we argue that the observed changes in the meridional extent of planetary-wave meanders are smaller than those implied by FV12. However, both studies agree on the sign of the meridional amplitude trends over NAmAtl, if not their magnitude or statistical significance.

\section{Conclusions}

[23] We have analyzed trends from 1979 to 2011 in two different measures of atmospheric planetary-wave amplitude: the meridional extent of meandering and the height of mid-tropospheric ridges and troughs at $45^{\circ} \mathrm{N}$. These two different measures of amplitude show notably different trends, and in many cases the trends are of opposing sign. Therefore, we argue that possible connections between AA and planetary waves, and implications of these, are sensitive to how waves are conceptualized. We find that statistically significant changes in either metric are limited to a few seasons, wavelengths, and longitudinal sectors. However, over the European-Atlantic region in summer, we observe significant, but opposing, meridional and zonal amplitude trends. These contrasting trends have different and complex possible implications for European summer weather, which further work will seek to address.

[24] Acknowledgments. The authors thank Jennifer Francis for helpful discussions and two anonymous reviewers for their comments. We gratefully acknowledge the ECMWF for providing on-line access to the ERA-Interim data. Parts of this research were funded by the Australian Research Council and the UK Natural Environment Research Council grant NE/J019585/1.

\section{References}

Buehler, T., C.C. Raible and T.F. Stocker (2011), The relationship of winter season North Atlantic blocking frequencies to extreme cold or dry spells in the ERA-40, Tellus, 63A, 212-222.

Cattiaux, J., R. Vautard, C. Cassou, P. Yiou, V. Masson-Delmotte and F. Codron (2010), Winter 2010 in Europe: A cold extreme in a warming climate, Geophys. Res. Lett., 37, L20704, doi:10.1029/2010GL044613.

Coumou, D. and S. Rahmstorf (2012), A decade of weather extremes, Nat. Clim. Change, 2,491-496.

Dee, D., et al. (2011) The ERA-Interim reanalysis: configuration and performance of the data assimilation system, Q. J. R. Meteorol. Soc., 137, 553-597.

Francis, J. A. and S. J. Vavrus (2012), Evidence linking Arctic amplification to extreme weather in mid-latitudes, Geophys. Res. Lett., 39, L06801, doi:10.1029/2012GL051000.

Guirguis, K., A. Gershunov, R. Schwartz and S. Bennett (2011), Recent warm and cold daily winter temperature extremes in the Northern Hemisphere, Geophys. Res. Lett., 38, L17701, doi:10.1029/2011GL048762.

Honda, M., J. Inoue and S. Yamane (2009), Influence of low Arctic sea-ice minima on anomalously cold Eurasian winters, Geophys. Res. Lett., 36. , L08707 doi:10.1029/2008GL037079.

Hopsch, S., J. Cohen and K. Dethloff (2012), Analysis of a link between fall Arctic sea ice concentration and atmospheric patterns in the following winter, Tellus, 64A, 18624, doi:10.3402/tellusa.v64i0.18624.

Inoue, J., M.E. Hori, K. Takaya (2012), The role of Barents sea ice in the wintertime cyclone track and emergence of a warm-Arctic cold-Siberian anomaly, J. Climate, 25, 2561-2568.

Liu, J., J.A. Curry, H. Wang, M. Song and R.M. Horton (2012), Impact of declining Arctic sea ice on winter snowfall. Proc. Natl. Acad. Sci. USA, 109, 4074-4079, doi:10.1073/pnas.1114910109.

Overland, J.E., K.R. Wood and M. Wang (2011), Warm Arctic - cold continents: climate impacts of the newly open Arctic sea, Polar Res., 30, 15787, doi:10.3402/polar.v30i0.15787.

Petoukhov, V. and V. Semenov (2010.) A link between reduced BarentsKara sea ice and cold winter extremes over northern continents, J. Geophys. Res., 115, D21111 doi:10.1029/2009JD013568.

Pfahl, S. and H. Wernli (2012), Quantifying the relevance of atmospheric blocking for co-located temperature extremes in the Northern Hemisphere on (sub-)daily time scales, Geophys. Res. Lett., L12807, doi:10.1029/ 2012GL052261.

Schubert, S., H. Wang, M. Suarez (2011), Warm season subseasonal variability and climate extremes in the Northern Hemisphere: The role of stationary Rossby waves, J. Climate, 24, 4773-4792. 


\section{SCREEN AND SIMMONDS: ARCTIC WARMING AND MID-LATITUDE WEATHER}

Screen, J.A. and I. Simmonds (2010), The central role of diminishing sea ice in recent Arctic temperature amplification, Nature, 464, $1334-1337$

Screen, J.A., C. Deser, I. Simmonds (2012), Local and remote controls on observed Arctic warming, Geophys. Res. Lett., 39, L10709, doi:10.1029/ 2012GL051598.

Screen, J.A., I. Simmonds, C. Deser and R. Tomas (2013), The atmospheric response to three decades of observed Arctic sea ice loss, J. Climate, 10.1175/JCLI-D-12-00063.1.
Serreze, M.C. and R.G. Barry (2011), Processes and impacts of Arctic amplification: A research synthesis, Global Planet. Change, $77,85-96$.

Sillmann, J., M. Corci-Maspoli, M. Kallache and R.W. Katz (2011), Extreme cold winter temperatures in Europe under the influence of North Atlantic atmospheric blocking, J. Climate, 24, 5899-5913. Solomon, S., D. Qin, M. Manning, Z. Chen, M. Marquis, K. Averyt, M. Tignot, and H. Miller (2007), Climate Change 2007: the Physical Science Basis, Cambridge University Press, Cambridge, UK. 